\title{
A Stochastic User Equilibrium Formulation for the Cumulative Prospect Theory-Based Cross-Nested Logit
}

\author{
Dongmei Yan $\left.{ }^{1}\right)^{1}$ and Yang Yang ${ }^{2}$ \\ ${ }^{1}$ Intelligent Transportation System Research Center, Southeast University, Nanjing 211189, China \\ ${ }^{2}$ School of Traffic and Logistics Engineering, Taiyuan University of Science and Technology, Taiyuan 030024, China
}

Correspondence should be addressed to Dongmei Yan; ydm_1988@163.com

Received 1 April 2021; Accepted 4 June 2021; Published 15 June 2021

Academic Editor: Shiping Wen

Copyright (c) 2021 Dongmei Yan and Yang Yang. This is an open access article distributed under the Creative Commons Attribution License, which permits unrestricted use, distribution, and reproduction in any medium, provided the original work is properly cited.

\begin{abstract}
The cumulative prospect theory provides a better description for route choice behavior of the travelers in an uncertain road network environment. In this study, we proposed a multiclass cumulative prospect value- (CPV-) based cross-nested logit (CNL) stochastic user equilibrium (SUE) model. For this model, an equivalent variational inequality (VI) model is provided, and the existence and equivalence of the model solutions are also proved. The method of successive averages (MSA), method of successive weighted averages (MSWA), and self-regulated averaging (SRA) method are designed and compared. In addition, the proposed multiclass CPV-based CNL SUE model is also compared with the multiclass utility value- (UV-) based CNL SUE model. The results show that the path flow assigned by the multiclass CPV-based CNL SUE model is more consistent with the actual situation. The impact of different model parameters on the cumulative prospect value (CPV) is investigated.
\end{abstract}

\section{Introduction}

Traffic assignment is a foundational problem in transportation research. Stochastic user equilibrium (SUE) model is an important method of traffic assignment. The route choice model is the key of the SUE model. The multinomial logit (MNL) model is typically used in the route choice model due to its simple mathematical structure. However, the MNL model has the property of independence of irrelevant alternatives (IIA) [1], that is, it does not consider the similarities between different paths. To this end, the crossnested logit (CNL) model is developed to deal with this problem [2]. At present, the SUE model based on the CNL route choice has been studied. Bekhor et al. [3] proposed a general CNL-SUE model. On this basis, Bekhor et al. [4] proposed a path-based algorithm for solving the CNL-SUE model and tested the performance of the algorithm. Along the same line, Du et al. [5] developed a path-based traffic assignment algorithm to solve the CNL SUE model by using the Barzilai-Borwein (BB) step size. In addition, $\mathrm{Hu}$ and Zhou [6] proposed a multiuser multimode CNL SUE model.
The CNL model is usually modeled based on expected utility theory (EUT). However, under uncertain road network environment, route choice behavior of travelers usually violates the EUT [7]. EUT assumes that travelers are perfectly rational when choosing a path. In reality, the route choice behavior of travelers is affected by environmental factors and psychological state. Therefore, cumulative prospect theory $(\mathrm{CPT})[8,9]$ is further developed. The CPT has been widely used in the process of traffic assignment modeling. Lo et al. [10] and Shao et al. [11], respectively, consider stochastic demand and link capacity degradation to establish travel time reliability models. On this basis, Siu and Lo [12] proposed a methodology to model the doubly uncertain transportation network with stochastic link capacity degradation and stochastic demand. Connors and Sumalee [13] provided a general modeling framework to describe the route choice behavior of travelers under uncertain travel time. $\mathrm{Xu}$ et al. [14] introduced the endogenous reference point to the UE model based on cumulative prospect theory. Wang and Sun [15] encapsulated the stochastic perception error (SPE) of travelers into the UE model. Yang and Jiang 
[16] extend the model in [15] to develop a CPT-based SUE model.

At present, there is no research on embedding the cumulative prospect theory into the CNL model framework in the SUE modeling process. Thus, the objective of this study is to propose a multiclass cumulative prospect value (CPV-) based CNL SUE model through using CPV replacing utility value (UV) as path performance. The equivalent variational inequality (VI) model and associated solution algorithm are provided. The Nguyen-Dupuis network is selected to compare the path flow assigned by the multiclass CPV-based CNL SUE model and the multiclass UV-based CNL SUE model. The computational efficiency of the three algorithms, i.e., the method of successive averages (MSA) [17], the method of successive weighted averages (MSWA) [18], and the self-regulated averages (SRA) [19], are also compared. The influence of model parameters on the CPV is analyzed.

The organization of this study is as follows. First, the multiclass CPV-based CNL SUE model is proposed. Then, the equivalent VI model and associated solution algorithms are provided. Afterwards, the numerical examples are investigated to demonstrate the performance of the proposed multiclass CPV-based CNL SUE model. Finally, conclusions of the study are provided.

\section{Study Model}

2.1. CPV-Based CNL Route Choice Model. It is well-known that traditional CNL route choice model is developed based on EUT. However, EUT usually exhibits inconsistency with traveler's route choice behavior in an uncertain traffic environment. Therefore, we use CPV instead of the utility value as path performance in the CNL framework to establish a more realistic route choice model. The steps of the CPVbased CNL route choice modeling are as follows:

First, the bureau of public roads (BPR) function is used to calculate the link travel time, namely,

$$
T_{a}\left(x_{a}, C_{a}\right)=t_{a}^{0}\left[1+\alpha\left(\frac{x_{a}}{C_{a}}\right)^{\beta}\right], \quad \forall a,
$$

where $T_{a}$ is the travel time on link $a, t_{a}^{0}$ and $C_{a}$ are the freeflow travel time and actual capacity on link $a$, respectively, and $\alpha$ and $\beta$ are deterministic parameters.

In reality, some events (e.g., natural disasters and traffic accidents) will cause link capacity to decrease, so that the link capacity becomes a random variable. Assume that the link capacity $C_{a}$ is uniformly distributed in the interval $\left[\zeta_{a} \bar{c}_{a}, \bar{c}_{a}\right]$ [10]. Since $C_{a}$ is a random variable, $T_{k i}^{w}$ is also a random variable. According to Lo et al. [10], the mean and variance of path travel time are given as follows:

$$
\begin{aligned}
& E\left(T_{k i}^{w}\right)=\sum_{a}\left\{\delta_{a, k}^{w}\left[t_{a}^{0}+\alpha t_{a}^{0} x_{a}^{\beta} \frac{1-\zeta_{a}^{1-\beta}}{\bar{c}_{a}^{\beta}\left(1-\zeta_{a}\right)(1-\beta)}\right]\right\}, \quad \forall w, k, i, \\
& \operatorname{var}\left(T_{k i}^{w}\right)=\sum_{a}\left\{\delta_{a, k}^{w} \alpha^{2}\left(t_{a}^{0}\right)^{2} x_{a}^{2 \beta}\left[\frac{1-\zeta_{a}^{1-2 \beta}}{\bar{c}_{a}^{2 \beta}\left(1-\zeta_{a}\right)(1-2 \beta)}-\left(\frac{1-\zeta_{a}^{1-\beta}}{\bar{c}_{a}^{\beta}\left(1-\zeta_{a}\right)(1-\beta)}\right)^{2}\right]\right\}, \quad \forall w, k, i, \\
& u_{0 i}^{w}=\min _{k}\left\{\mathscr{B}_{k i}^{w}\right\}, \quad \forall w, k, i,
\end{aligned}
$$

where $\bar{c}_{a}$ is the design capacity of link $a, \zeta_{a} \bar{c}_{a}$ is the worstdegraded capacity, $\zeta_{a}$ is a fraction of the design capacity, and $\delta_{a, k}^{w}$ represents the relationship between path $k$ and link $a$ in $\mathrm{O}$-D pair $w$. Note that $\delta_{a, k}^{w}=1$ if link $a$ is on path $k ; \delta_{a, k}^{w}=0$, otherwise.

Second, the endogenous reference point proposed by $\mathrm{Xu}$ et al. [14] is adopted here. In other words, the minimum travel time budget is defined as the reference point. The expression of the reference point is as follows:

$$
\begin{gathered}
P\left\{T_{k i}^{w} \leq \mathscr{B}_{k i}^{w}=E\left(T_{k i}^{w}\right)+\xi_{i} \sigma_{k i}^{w}\right\}=\rho_{i}, \quad \forall w, k, i, \\
\mathscr{B}_{k i}^{w}=E\left(T_{k i}^{w}\right)+\sigma_{k i}^{w} \Phi^{-1}\left(\rho_{i}\right), \quad \forall w, k, i,
\end{gathered}
$$

where $\mathscr{B}_{k i}^{w}$ is the travel time budget of user class $i$ on path $k$ between O-D pairs $w, E\left(T_{k i}^{w}\right)$ and $\sigma_{k i}^{w}$ are the mean and standard deviation of $T_{k i}^{w}$, respectively, $\rho_{i}$ is the on-time arrival probability of user class $i, \Phi^{-1}$ is the inverse function of the standard normal cumulative distribution function, $u_{0 i}^{w}$ is the reference point of user class $i$ between O-D pairs $w$, and $\xi_{i}$ is a parameter related to on-time arrival probability of user class $i$.

Third, the CPV of the path is calculated by the value function, and the probability weight function is as follows:

$$
\begin{aligned}
& g\left(T_{k i}^{w}\right)=\left\{\begin{array}{cc}
\left(u_{0 i}^{w}-T_{k i}^{w}\right)^{\delta}, & T_{k i}^{w} \leq u_{0 i}^{w}, \\
-\lambda\left(T_{k i}^{w}-u_{0}^{w}\right)^{\eta}, & T_{k i}^{w}>u_{0 i}^{w},
\end{array} \quad \forall w, k, i,\right.
\end{aligned}
$$




$$
v_{k i}^{w}=\int_{\bar{u}_{k i}^{w}}^{u_{0 i}^{w}} \frac{\mathrm{d} \omega^{+}\left[F\left(T_{k i}^{w}\right)\right]}{\mathrm{d} T_{k i}^{w}} g\left(T_{k i}^{w}\right) \mathrm{d} T_{k i}^{w}+\int_{u_{0 i}^{w}}^{\bar{u}_{k i}^{w}}-\frac{\mathrm{d} \omega^{-}\left[1-F\left(T_{k i}^{w}\right)\right]}{\mathrm{d} T_{k i}^{w}} g\left(T_{k i}^{w}\right) \mathrm{d} T_{k i}^{w}, \quad \forall w, k, i,
$$

where $\delta$ and $\eta$ are the degrees of diminishing sensitivity, $0<\delta, \eta<1, \lambda$ is the loss-aversion coefficient, $\lambda \geq 1$, and $\gamma$ is the degree of curvature of the probability weight function, $0<\gamma<1$. In addition, $\omega(p)$ and $p$ are the perceived probability and actual probability of the event, respectively. Note that $\omega(p)$ increases monotonously with respect to $p$, and it has nothing to do with the value of $\gamma . F\left(T_{k i}^{w}\right)$ is the distribution function of $T_{k i}^{w}$, and $\omega^{+}$and $\omega^{-}$are the gain area and loss area of probability weighting function, respectively. In equation (9), $\underline{u}_{k, i}^{w}$ and $\bar{u}_{k, i}^{w}$ are the lower bound and upper bound of the travel time of user class $i$ on path $k$ between $\mathrm{O}-\mathrm{D}$ pairs $w$, respectively. The lower bound is free flow time, and the upper bound is $\bar{u}_{k i}^{w}=E\left[\left(T_{k i}^{w}\right)\right]+3 \sqrt{\operatorname{var}\left(T_{k i}^{w}\right)}$.

Finally, we apply the CPV instead of the utility value as the path performance in the CNL framework. The probability of user class $i$ choosing path $k$ between O-D pairs $w$ is

$$
P_{k i}^{w}=\frac{\exp \left[\theta_{i} v_{k i}^{w}+\ln \sum_{m \in M} \alpha_{m k}^{w}\left(\sum_{l} \alpha_{m l}^{w} \exp \left(\theta_{i} v_{l i}^{w}\right)\right)^{\mu_{m}^{w}-1}\right]}{\sum_{j \in K_{w}} \exp \left[\theta_{i} v_{j i}^{w}+\ln \sum_{m \in M} \alpha_{m j}^{w}\left(\sum_{l} \alpha_{m l}^{w} \exp \left(\theta_{i} v_{l i}^{w}\right)\right)^{\mu_{m}^{w}-1}\right]}, \quad \forall w, k, i
$$

where $k, j$, and $l$ are the path indicators, $v_{l i}^{w}$ is the CPV of user class $i$ on path $l$ between O-D pairs $w, \theta_{i}$ is the dispersion parameter of user class $i, \mu_{m}^{w}$ is the nesting coefficient, $0<\mu_{m}^{w} \leq 1$, and $e_{m k}^{w}$ is the inclusion coefficient. According to Prashker and Bekhor [20], $e_{m k}^{w}=\left(L_{m} / L_{k}\right)^{\tau} \delta_{m k}^{w}$ and $\mu_{m}^{w}=1-\left(1 / G_{m}^{w}\right) \sum_{k} e_{m k}^{w}$, where $L_{m}$ is the length of link $m, L_{k}$ is the length of path $k, G_{m}^{w}$ is the number of paths including link $m$ between O-D pairs $w$, and $\tau$ is a parameter that characterizes the travelers' perception of the similarities between different paths, $\tau=1$.

It is possible to decompose equation (10) as marginal probability and conditional probability, that is,

$$
P_{k i}^{w}=\sum_{m} P_{i}^{w}(m) P_{i}^{w}(k \mid m), \quad \forall w, k, i .
$$

The marginal probability is described as

$$
P_{i}^{w}(m)=\frac{\left(\sum_{k}\left(e_{m k}^{w} \exp \left(\theta_{i} v_{k i}^{w}\right)\right)^{1 / \mu_{m}^{w}}\right)^{\mu_{m}^{w}}}{\sum_{m}\left(\sum_{k}\left(e_{m k}^{w} \exp \left(\theta_{i} v_{k i}^{w}\right)\right)^{1 / \mu_{m}^{w}}\right)^{\mu_{m}^{w}}}, \quad \forall w, m, i
$$

and the conditional probability is described as

$$
P_{i}^{w}(k \mid m)=\frac{\left(e_{m k}^{w} \exp \left(\theta_{i} v_{k i}^{w}\right)\right)^{1 / \mu_{m}^{w}}}{\sum_{l}\left(e_{m l}^{w} \exp \left(\theta_{i} v_{l i}^{w}\right)\right)^{1 / \mu_{m}^{w}}}, \quad \forall w, k, m, i
$$

2.2. Multiclass CPV-Based CNL SUE Model. According to the SUE principle, the network equilibrium state satisfies the following condition:

$$
\sum_{m} f_{m k i}^{w}=q_{i}^{w} P_{k i}^{w}, \quad \forall w, k, i .
$$

The regular constraints must hold, that is,

$$
\begin{aligned}
q_{i}^{w} & =\sum_{m} \sum_{k} f_{m k i}^{w}, \quad \forall w, i, \\
x_{a i} & =\sum_{w} \sum_{k} \sum_{m} f_{m k i}^{w} \delta_{a k}^{w}, \quad \forall a, i, \\
f_{m k i}^{w} & \geq 0, \quad \forall w, k, m, i,
\end{aligned}
$$

where $q_{i}^{w}$ is the travel demand of user class $i$ between O-D pairs $w, f_{m k i}^{w}$ is the flow of user class $i$ on path $k$ of nest $m$ between O-D pairs $w$, and $x_{a i}$ is the flow of user class $i$ on link $a$.

\section{Equivalent VI Model and Solution Algorithm}

3.1. Equivalent VI Model. The solution of the multiclass CPV-based CNL SUE model is equivalent to seeking a feasible path flow, so that the following variational inequality (VI) model holds

$$
\sum_{w} \sum_{m} \sum_{k} \sum_{i}\left[\frac{1-\mu_{m}^{w}}{\theta_{i}} \ln \left(\sum_{k} f_{m k i}^{w *}\right)+\frac{\mu_{m}^{w}}{\theta_{i}} \ln f_{m k i}^{w *}-\frac{1}{\theta_{i}} \ln \left(e_{m k}^{w}\right)-v_{k i}^{w}\right] \times\left(f_{m k i}^{w}-f_{m k i}^{w *}\right) \geq 0 .
$$

\subsection{Equivalence and Existence}

(1) Equivalence 
According to the VI theorem, equation (18) can be written as the following equivalent complementary relaxation condition:

$$
\begin{array}{r}
\left(\frac{1-\mu_{m}^{w}}{\theta_{i}} \ln \left(\sum_{k} f_{m k i}^{w *}\right)+\frac{\mu_{m}^{w}}{\theta_{i}} \ln f_{m k i}^{w *}-\frac{1}{\theta_{i}} \ln \left(e_{m k}^{w}\right)-v_{k i}^{w}\right) \times f_{m k i}^{w *}=0, \\
\left(\frac{1-\mu_{m}^{w}}{\theta_{i}} \ln \left(\sum_{k} f_{m k i}^{w *}\right)+\frac{\mu_{m}^{w}}{\theta_{i}} \ln f_{m k i}^{w *}-\frac{1}{\theta_{i}} \ln \left(e_{m k}^{w}\right)-v_{k i}^{w}\right) \geq 0 .
\end{array}
$$

When $f_{m k i}^{w *}>0$, we have

$$
\frac{1-\mu_{m}^{w}}{\theta_{i}} \ln \left(\sum_{k} f_{m k i}^{w *}\right)+\frac{\mu_{m}^{w}}{\theta_{i}} \ln f_{m k i}^{w *}-\frac{1}{\theta_{i}} \ln \left(e_{m k}^{w}\right)-v_{k i}^{w}=0 .
$$

After a series of transformations, the marginal probability is obtained as follows:

$P_{i}^{w}(m)=\frac{\sum_{k} f_{m k i}^{w *}}{\sum_{m} \sum_{k} f_{m k i}^{w *}}=\frac{\left\{\sum_{k}\left(e_{m k}^{w}\right)^{\left(1 / \mu_{m}^{w}\right)} \exp \left[\left(\theta_{i} v_{k i}^{w}\right) / \mu_{m}^{w}\right]\right\}^{\mu_{m}^{w}}}{\sum_{m}\left\{\sum_{k}\left(e_{m k}^{w}\right)^{\left(1 / \mu_{m}^{w}\right)} \exp \left[\left(\theta_{i} v_{k i}^{w}\right) / \mu_{m}^{w}\right]\right\}^{\mu_{m}^{w}}}$.

And then, the conditional probability is also obtained as follows:

$$
P_{i}^{w}(k \mid m)=\frac{f_{m k i}^{w *}}{\sum_{k} f_{m k i}^{w *}}=\frac{\left(e_{m k}^{w}\right)^{\left(1 / \mu_{m}^{w}\right)} \exp \left[\left(\theta_{i} v_{k i}^{w}\right) / \mu_{m}^{w}\right]}{\sum_{k}\left(e_{m k}^{w}\right)^{\left(1 / \mu_{m}^{w}\right)} \exp \left[\left(\theta_{i} v_{k i}^{w}\right) / \mu_{m}^{w}\right]}
$$

Obviously, equations (21) and (22) correspond to equations (12) and (13). Thus, the solution of the variational inequality model in equation (18) is equivalent to the solution of the multiclass CPVbased CNL SUE model.

\section{(2) Existence}

Let $F\left(f_{m k i}^{w}\right)=\left(\left(1-\mu_{m}^{w}\right) / \theta_{i}\right) \ln \left(\sum_{k} f_{m k i}^{w}\right)+\left(\mu_{m}^{w} / \theta_{i}\right) \ln$ $f_{m k i}^{w}-\left(1 / \theta_{i}\right) \ln \left(e_{m k}^{w}\right)-v_{k i}^{w}$. The distribution function of link travel time is assumed to be a continuous function of the link flow. According to equations
(4)-(6), the reference point is a continuous function of the path flow. According to equations (7)-(9), the CPV of the path is a continuous function of the path flow. Thus, $F\left(f_{m k i}^{w}\right)$ is also a continuous function of the path flow. In addition, according to equations (15)-(17), the set of feasible path flow is a compact convex set. Based on the VI theorem, the solution of equation (18) exists.

3.3. Solution Algorithm. The SUE model is usually solved by the method of the successive averages (MSA) algorithm. However, the predetermined iterative step length causes the convergence speed of the MSA algorithm to be very slow. Therefore, we apply the MSA algorithm, MSWA algorithm, and SRA algorithm to solve the equivalent VI model and compare the computational efficiency of these three algorithms. The calculation steps of these three algorithms are as follows:

Step 1. Initialization.

Determine the feasible path set $K_{w}$. Calculate the reference point and the $\mathrm{CPV}$ based on zero flow. According to equation (14), the initial path flow pattern is obtained by assigning the O-D demand to the link. Set iteration counter $n=1$.

Step 2. Update the CPV of the path.

Recalculate the mean and variance of the path travel time based on equations (2) and (3). For each O-D pair, redetermine the reference point according to equations (4)-(6). Update the CPV of each path according to equation (9).

Step 3. Direction finding.

Based on the updated CPV of the path, reassign the O-D demand to the link to obtain the auxiliary path flow $\mathbf{g}^{(n)}$, and then, iterative direction $\mathbf{g}^{(n)}-\mathbf{f}^{(n)}$ is also obtained.

Step 4. Update path flow.

Set $\mathbf{f}^{(n+1)}=\mathbf{f}^{(n)}+\chi^{(n)}\left(\mathbf{g}^{(n)}-\mathbf{f}^{(n)}\right)$. For the MSA algorithm, $\chi^{(n)}=(1 / n)$. For the MSWA algorithm, $\chi^{(n)}=\left(n^{d} /\left(1^{d}+2^{d}+3^{d}+\cdots+n^{d}\right)\right), d \geq 0$. For the SRA algorithm, $\chi^{(n)}=\left(1 / \mathscr{H}^{n}\right)$, where

$$
\mathscr{H}^{n}= \begin{cases}\mathscr{H}^{(n-1)}+\Gamma, \Gamma>1, & \text { if }\left\|\mathbf{f}^{(n)}-\mathbf{g}^{(n)}\right\| \geq\left\|\mathbf{f}^{(n-1)}-\mathbf{g}^{(n-1)}\right\|, \\ \mathscr{H}^{(n-1)}+L, L<1, & \text { if }\left\|\mathbf{f}^{(n)}-\mathbf{g}^{(n)}\right\|<\left\|\mathbf{f}^{(n-1)}-\mathbf{g}^{(n-1)}\right\|,\end{cases}
$$

Step 5. Convergence test
If $\left\|\mathbf{f}^{(n)}-\mathbf{g}^{(n)}\right\| \leq \varepsilon$, then stop. If not, set $n=n+1$ and go to Step 2. 


\section{Numerical Examples}

In this section, we first compare the computational efficiency of the MSA algorithm, MSWA algorithm, and SRA algorithm. Then, we compare the path flow assigned by the multiclass CPV-based CNL SUE model and the multiclass UV-based CNL SUE model. Finally, we analyze the influence of different model parameters on the CPV of path.

4.1. Preliminary. The Nguyen-Dupuis network is selected to test the performance of the proposed multiclass CPV-based CNL SUE model. The Nguyen-Dupuis network includes 13 nodes and 19 links, as shown in Figure 1. For each O-D pair, the travel demand is $q_{1-2}=660, q_{1-3}=495, q_{4-2}=412.5$, and $q_{4-3}=495$, respectively.

The free-flow time, design capacity, and the worst-degraded coefficient are given in Table 1, and the path composition and path length are given in Table 2. It should be noted that the values in Tables 1 and 2 are adopted from studies by Xu et al. [14] and Jiang and Xie [21], respectively.

4.2. Comparison of Computational Efficiency among MSA, $M S W A$, and SRA. The three different algorithms (i.e., MSA, MSWA, and SRA) are implemented on the Nguyen-Dupuis network to compare their computational efficiency. According to Liu et al. [18], we set $\Gamma=1.9, \mathscr{L}=0.1$, and $d=2$. The computational efficiency of MSA, MSWA, and SRA is given in Table 3 .

In Table 3, it can be seen that the MSA algorithm may provide a faster convergence speed than the SRA algorithm and the MSWA algorithm at the initial stage. However, when the number of iterations gradually increases, the SRA achieves a preset accuracy with a fastest convergence speed in all algorithms. This is because the step size of the SRA algorithm will be adjusted according to the information provided in the two iterations. In contrast, the step size of the MSA algorithm is predetermined, and it is difficult to give an optimal weight parameter of the MSWA algorithm in advance. Therefore, the computational efficiency of the SRA algorithm outperforms the MSA algorithm and the MSWA algorithm.

4.3. Comparative Analysis of Path Flows Based on CPT and EUT. To demonstrate the performance of the proposed multiclass CPV-based CNL SUE model, the path flow assigned by the proposed model and the multiclass UVbased CNL SUE model are shown in Figure 2. Travelers are divided into four categories based on their familiarity with the conditions of the road network. The dispersion parameters and nesting coefficients of these four user classes are set to $\theta=\mu=0.25, \theta=\mu=0.5, \theta=\mu=0.75$, and $\theta=\mu=1$, respectively. In addition, the parameters in the BPR function are $\alpha=0.15$ and $\beta=4$, the on-time arrival probability is defined as $\rho=0.8$, and the iteration accuracy is set to $\varepsilon=0.0001$. According to the parameter calibration results of Kahneman and Tversky [22] and Prelec [23], the parameters in the value function are set to $\delta=\eta=0.88$ and

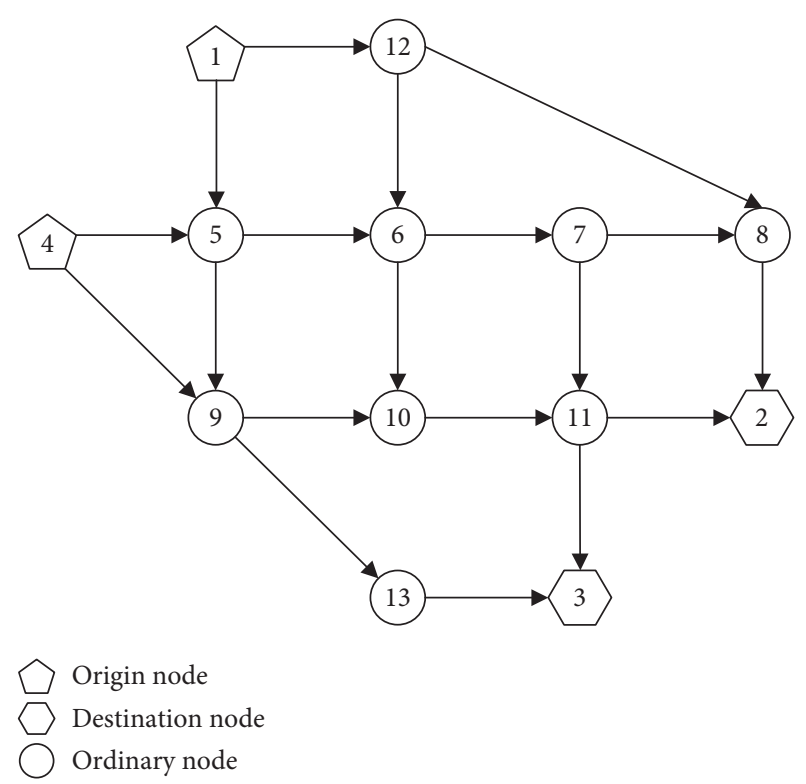

Figure 1: Nguyen-Dupuis network.

TABLE 1: Link characteristics.

\begin{tabular}{lcccc}
\hline No. & Link & $t_{a}^{0}$ & $\bar{c}_{a}$ & $\zeta_{a}$ \\
\hline 1 & $1-5$ & 7 & 300 & 0.8 \\
2 & $1-12$ & 9 & 200 & 0.8 \\
3 & $4-5$ & 9 & 200 & 0.7 \\
4 & $4-9$ & 12 & 200 & 0.8 \\
5 & $5-6$ & 3 & 350 & 0.6 \\
6 & $5-9$ & 9 & 400 & 0.6 \\
7 & $6-7$ & 5 & 500 & 0.7 \\
8 & $6-10$ & 13 & 250 & 0.8 \\
9 & $7-8$ & 5 & 250 & 0.7 \\
10 & $7-11$ & 9 & 300 & 0.7 \\
11 & $8-2$ & 9 & 500 & 0.7 \\
12 & $9-10$ & 10 & 550 & 0.6 \\
13 & $9-13$ & 9 & 200 & 0.8 \\
14 & $10-11$ & 6 & 400 & 0.7 \\
15 & $11-2$ & 9 & 300 & 0.7 \\
16 & $11-3$ & 8 & 300 & 0.6 \\
17 & $12-6$ & 7 & 200 & 0.8 \\
18 & $12-8$ & 14 & 300 & 0.7 \\
19 & $13-3$ & 11 & 200 & 0.7 \\
\hline
\end{tabular}

$\lambda=2.25$, and the parameter in the probability weight function is considered as $\gamma=0.74$.

In Figure 2, it can be seen that the path flow assigned by the proposed multiclass CPV-based CNL SUE model and the multiclass UV-based CNL SUE model is significantly different. This is because the proposed model assumes that travelers are bounded rationally when choosing a path, while the multiclass UV-based CNL SUE model is based on an assumption that travelers are perfectly rational when choosing a path.

It can also be seen from the path flow assigned by the multiclass CPV-based CNL SUE model that some travelers choose a path with a high mean travel time but low travel time standard deviation. For example, when $\theta=0.25$, the 
TABLe 2: Path composition and length.

\begin{tabular}{|c|c|c|c|}
\hline O-D & Path & Node sequence & Path length \\
\hline \multirow{8}{*}{$1-2$} & 1 & $1-12-8-2$ & 32 \\
\hline & 2 & $1-5-6-7-8-2$ & 29 \\
\hline & 3 & $1-5-6-7-11-2$ & 33 \\
\hline & 4 & $1-5-6-10-11-2$ & 38 \\
\hline & 5 & $1-5-9-10-11-2$ & 41 \\
\hline & 6 & $1-12-6-7-8-2$ & 35 \\
\hline & 7 & $1-12-6-7-11-2$ & 39 \\
\hline & 8 & $1-12-6-10-11-2$ & 44 \\
\hline \multirow{5}{*}{$4-2$} & 15 & $4-9-10-11-2$ & 37 \\
\hline & 16 & $4-5-6-7-8-2$ & 31 \\
\hline & 17 & $4-5-6-7-11-2$ & 35 \\
\hline & 18 & $4-5-6-10-11-2$ & 40 \\
\hline & 19 & $4-5-9-10-11-2$ & 43 \\
\hline \multirow{6}{*}{$1-3$} & 9 & $1-5-9-13-3$ & 36 \\
\hline & 10 & $1-5-6-7-11-3$ & 32 \\
\hline & 11 & $1-5-6-10-11-3$ & 37 \\
\hline & 12 & $1-5-9-10-11-3$ & 40 \\
\hline & 13 & $1-12-6-7-11-3$ & 38 \\
\hline & 14 & $1-12-6-10-11-3$ & 43 \\
\hline \multirow{6}{*}{$4-3$} & 20 & $4-9-13-3$ & 32 \\
\hline & 21 & $4-9-10-11-3$ & 36 \\
\hline & 22 & $4-5-9-13-3$ & 38 \\
\hline & 23 & $4-5-6-7-11-3$ & 34 \\
\hline & 24 & $4-5-6-10-11-3$ & 39 \\
\hline & 25 & $4-5-9-10-11-3$ & 42 \\
\hline
\end{tabular}

TABLE 3: Computational efficiency of MSA, MSWA, and SRA.

\begin{tabular}{lccc}
\hline $\begin{array}{l}\text { Number of iterations } \\
n\end{array}$ & \multicolumn{3}{c}{ Convergence criterion $\varepsilon^{k}=\mathbf{f}^{k}-\mathbf{g}^{k}$} \\
\hline 100 & MSA & MSWA & SRA \\
200 & 75.94 & 491.00 & 257.91 \\
400 & 24.31 & 348.15 & 22.24 \\
800 & 12.13 & 19.18 & 4.62 \\
1600 & 6.06 & 2.39 & 0.36 \\
\hline
\end{tabular}

mean travel time on path 5 is greater than that of path 6 , and the travel time standard deviation is smaller than that of path 6. More flow is assigned on path 5 than that of path 6 . In contrast, the path flow assigned by the multiclass UV-based CNL SUE model indicates that all travelers choose the path with a low mean travel time, regardless of the travel time standard deviation. Similarly, the path flow under other dispersion parameters and nesting coefficients shows a similar situation, so we will not repeat them here. Furthermore, some empirical studies [24, 25] have shown that travelers are bounded rationally when choosing a path. The path flow assigned by the proposed multiclass CPV-based CNL SUE model is aligned with the actual situation. Therefore, it can be concluded from the above analysis that the proposed multiclass CPV-based CNL SUE model can deal with perfectly rational issue.

4.4. Sensitivity Analysis. To analyze the influence of different model parameters on the CPV of the path, we select all paths in the O-D pair $(4,2)$. It should be noted that the colors in Figure 3 represent CPVs of paths.

In Figure 3, it can first be seen that the CPV hardly changes with the parameter $\mu$. This means that the parameter $\mu$ has no significant influence on the CPV. In addition, it can also be seen that the parameters $\rho, \zeta_{a}$, and $\delta$ have a great impact on the CPV, while the parameter $\theta$ has a small impact on the CPV.

Second, as the increase of the parameters $\theta, \rho, \delta$, and $\gamma$, the CPV of the path gradually increases. Specifically, when the parameter $\theta$ becomes larger, travelers know more road network information. At this time, the travelers' route choice tends to be deterministic, which will lead to a slight increase in the CPV of the path. When the parameter $\rho$ increases, the reference point becomes larger. This means that travelers increase their psychological expectations of the travel time standard. At this time, travelers' benefits increase, and the $\mathrm{CPV}$ of the path naturally increases. When the parameter $\delta$ becomes larger, the degree of diminishing sensitivity in the profit area decreases. When the path travel time is lower than the reference point, travelers may obtain greater benefits, so the CPV of the path increases. When the parameter $\gamma$ increases, travelers pay more attention to large-probability events. This means that the weight of high-probability events will increase. It is a high-probability event that travelers obtain benefits. So the CPV of the path increases.

Third, as the parameters $\lambda$ and $\eta$ increase, the CPV of the path decrease steadily. Specifically, when the parameter $\lambda$ becomes larger, the travelers pay more attention to the loss. The proportion of the loss part in the CPV will increase, so the CPV of the path decreases. When the parameter $\eta$ 


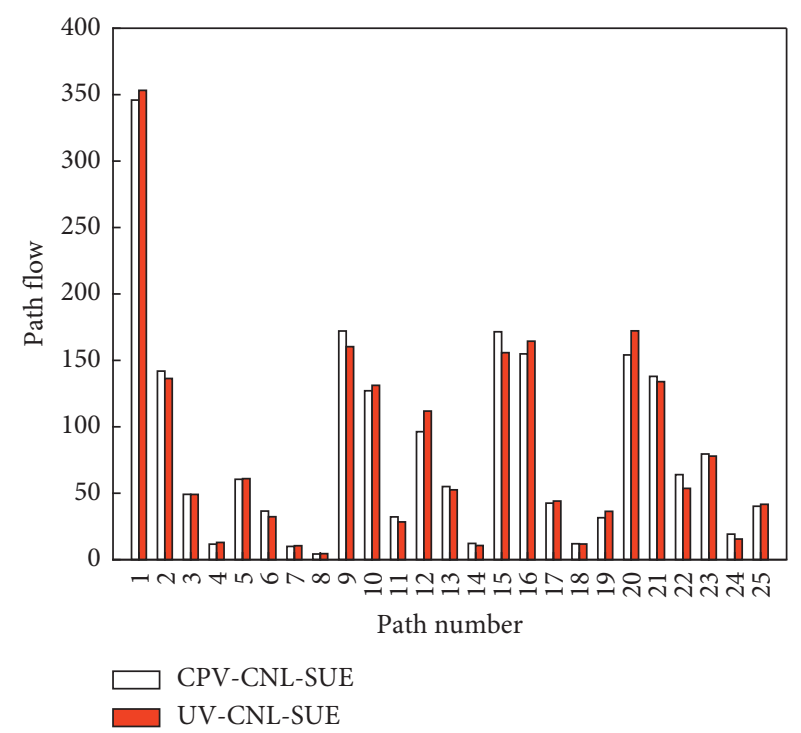

(a)

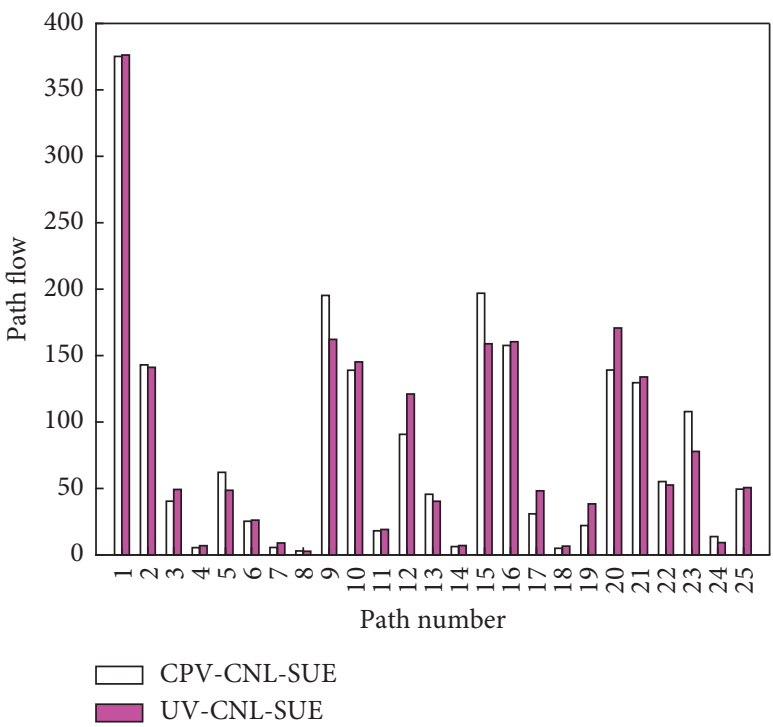

(c)

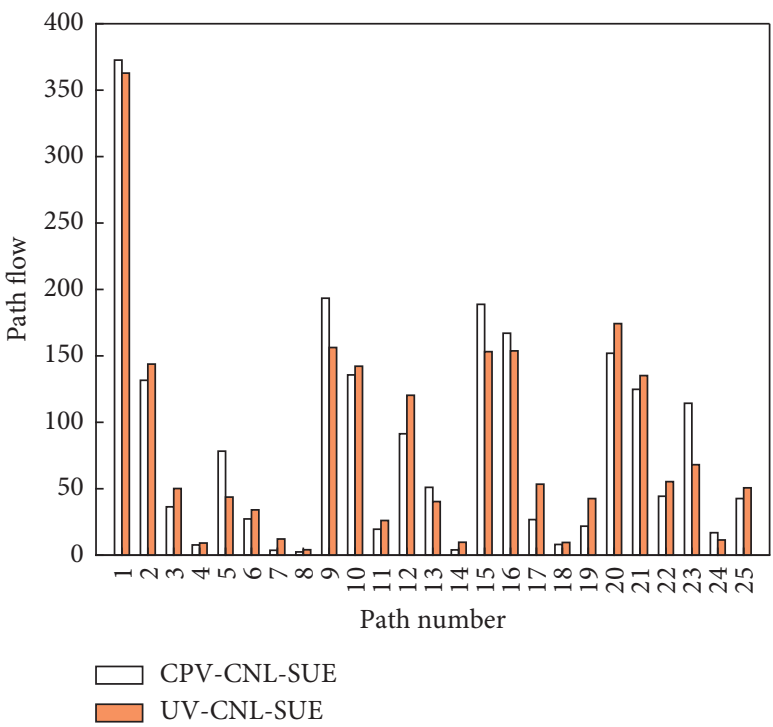

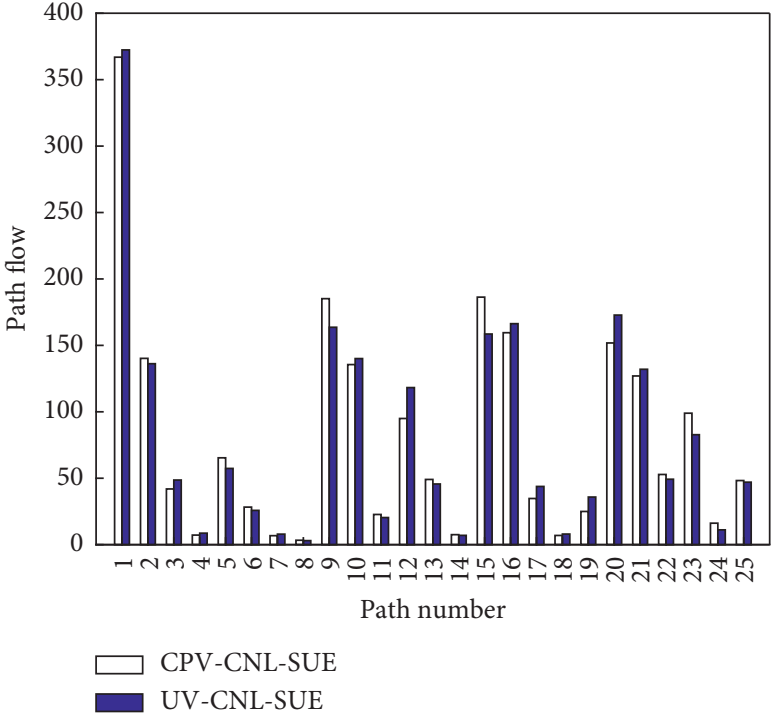

(b)

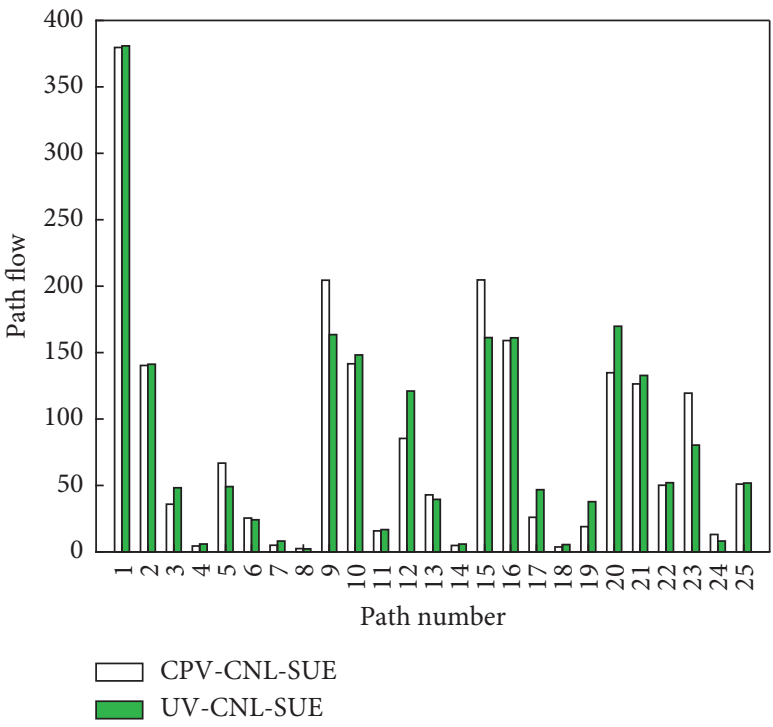

(d)

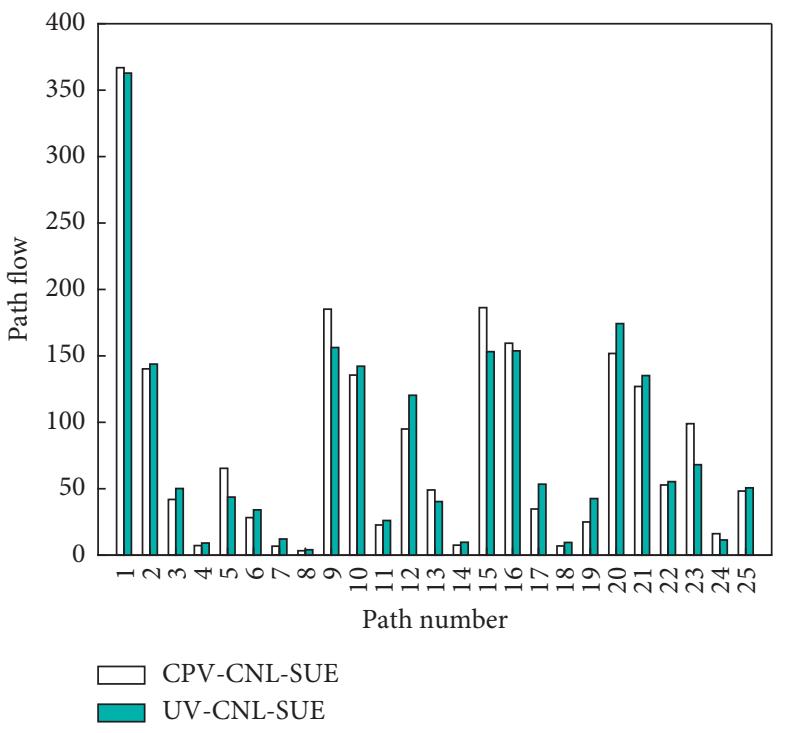

(f)

FIgure 2: Continued. 


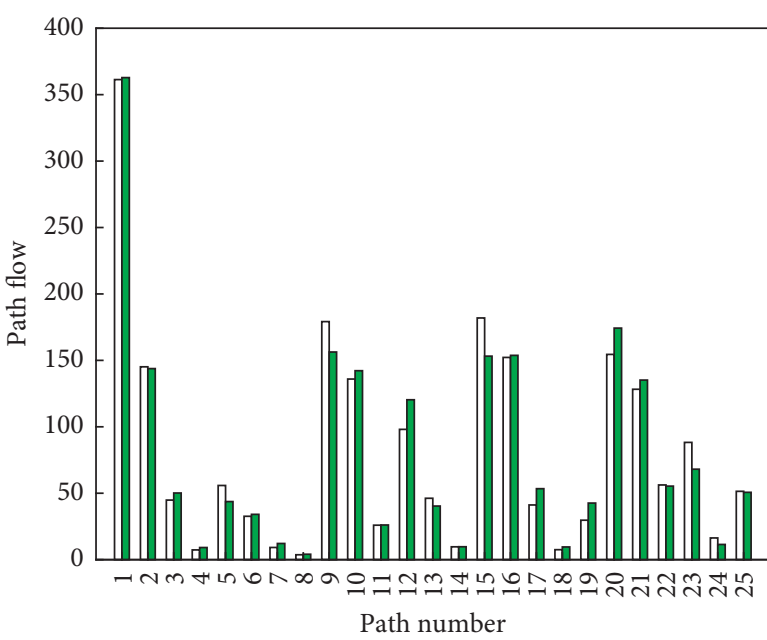

$\square$ CPV-CNL-SUE UV-CNL-SUE

(g)

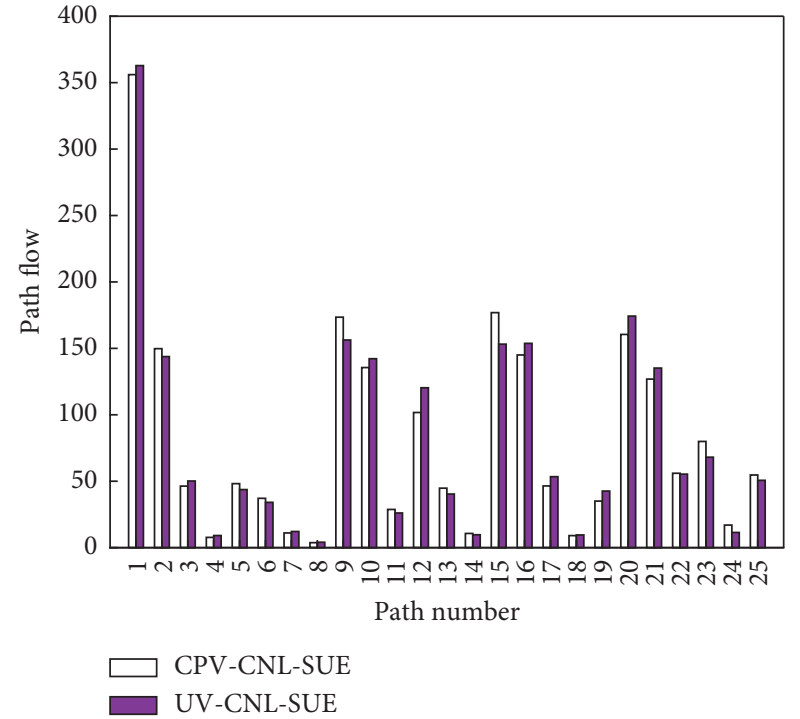

(h)

Figure 2: The path flow under different parameters. (a) $\theta=0.25$. (b) $\theta=0.5$. (c) $\theta=0.75$. (d) $\theta=1$. (e) $\mu=0.25$. (f) $\mu=0.5$. (g) $\mu=0.75$. (h) $\mu=1$.

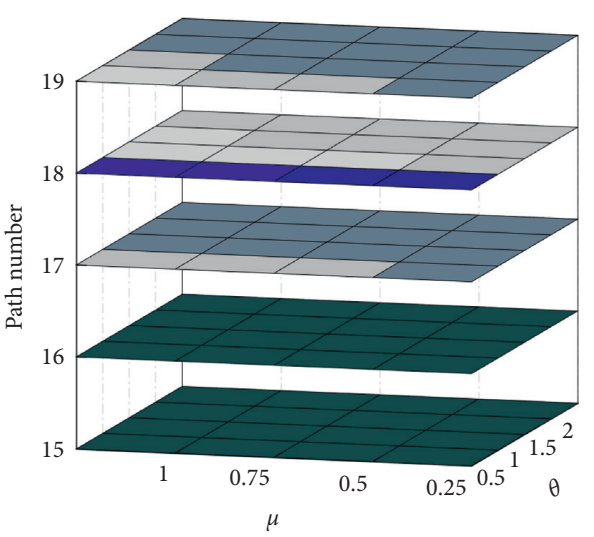

(a)

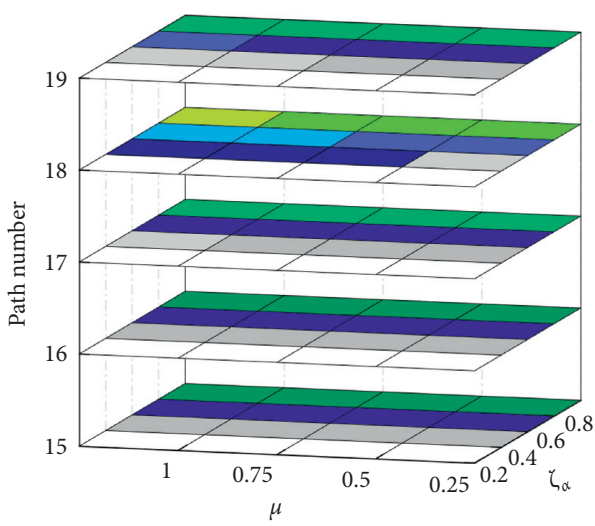

(c)
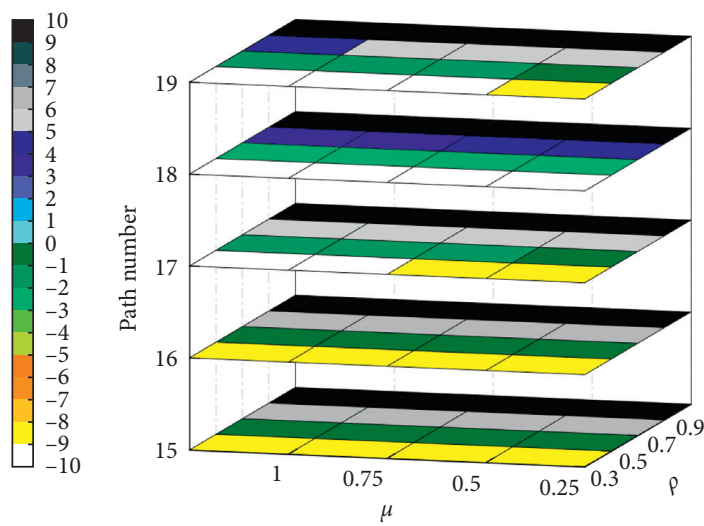

(b)
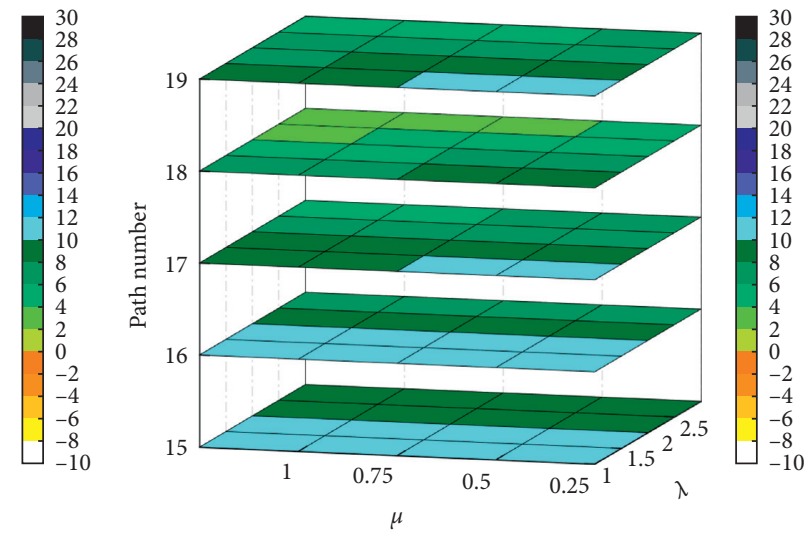

Figure 3: Continued. 


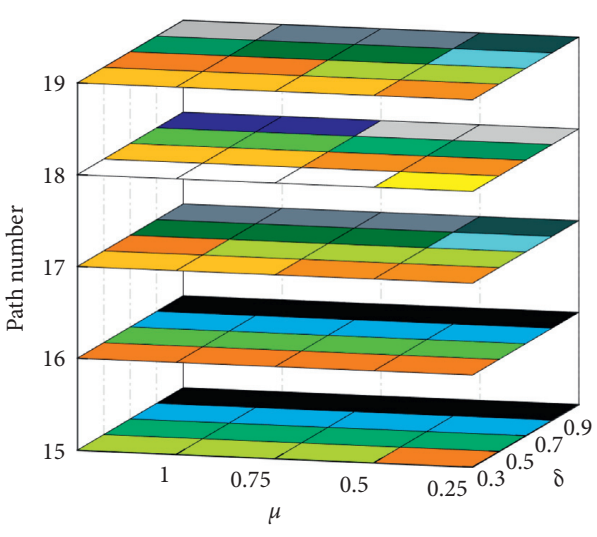

(e)
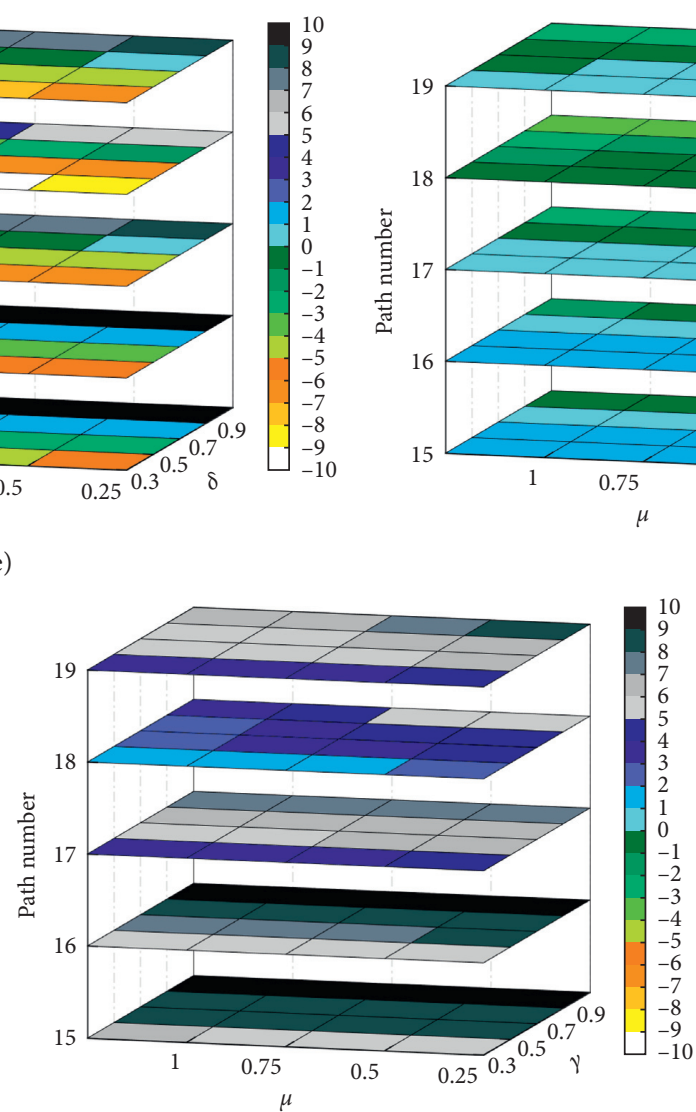

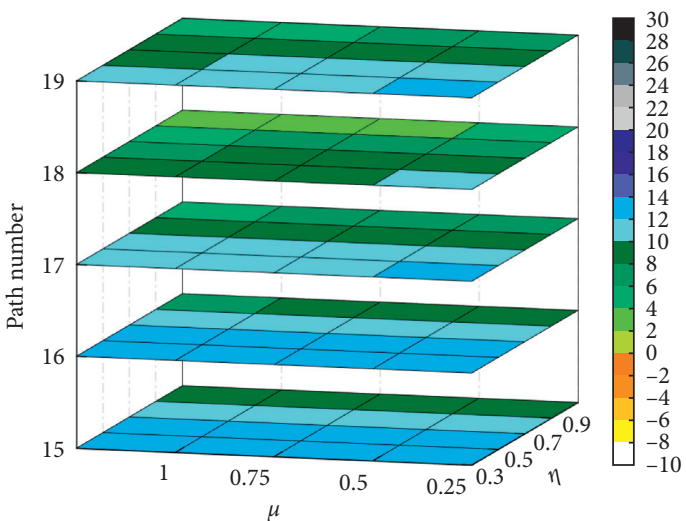

(f)

(g)

Figure 3: The variation of the CPV with model parameters. (a) Sensitivity analysis of $\mu$ and $\theta$. (b) Sensitivity analysis of $\mu$ and $\rho$. (c) Sensitivity analysis of $\mu$ and $\zeta_{a}$. (d) Sensitivity analysis of $\mu$ and $\lambda$. (e) Sensitivity analysis of $\mu$ and $\delta$. (f) Sensitivity analysis of $\mu$ and $\eta$. (g) Sensitivity analysis of $\mu$ and $\gamma$.

increases, the degree of diminishing sensitivity in the loss area decreases. When the path travel time is greater than the reference point, travelers may suffer more losses, so the CPV of the path decreases.

Finally, as the parameter $\zeta_{a}$ increases, the CPV of the path initially increases and then decreases. When the parameter $\zeta_{a}=0.2$, the link capacity is very low, so the CPV of the path is small. As the parameter $\zeta_{a}$ increases, the reliability of the link travel time increases. At this time, travelers' psychological expectations for travel time standards decrease, and travelers' losses will increase, so the CPV of the path will naturally decrease.

\section{Conclusion}

This study proposes a multiclass CPV-based CNL SUE model by using CPV instead of UV as path performance in the CNL framework. An equivalent variational inequality model is provided. Three solution algorithms (i.e., MSA, MSWA, and SRA) are designed and compared. The results show that the SRA algorithm has the highest computational efficiency. In addition, we compare the path flow assigned by the proposed multiclass CPV-based CNL SUE model and the multiclass UV-based CNL SUE model. The results indicate that the path flow assigned by the proposed model is more consistent with the actual route choice behavior of travelers. The influence of parameters on the CPV of the path is also investigated.

The model parameters have an important influence on the process of traffic assignment modeling. We will combine survey and experimental date to investigate calibration of model parameters in the future. In addition, travel demand exhibits elastic characteristics under the saturated traffic condition. We will extend fixed demand to elastic demand in future research.

\section{Data Availability}

The data used to support the findings of this study are included within the article and are cited at relevant places within the text as references.

\section{Conflicts of Interest}

The authors declare that there are no conflicts of interest.

\section{Acknowledgments}

This research was supported by the Postgraduate Research and Practice Innovation Program of Jiangsu Province (KYLX16_0271). 


\section{References}

[1] C. H. Wen and F. S. Koppelman, "The generalized nested logit model," Transportation Research Part B: Methodological, vol. 35, no. 7, pp. 627-641, 2001.

[2] S. Ghader, C. Carrion, L. Tang, A. Asadabadi, and L. Zhang, "A copula-based continuous cross-nested logit model for tour scheduling in activity-based travel demand models," Transportation Research Part B: Methodological, vol. 145, no. 3, pp. 324-341, 2021.

[3] S. Bekhor, L. Reznikova, and T. Toledo, "Application of crossnested logit route choice model in stochastic user equilibrium traffic assignment," Transportation Research Record: Journal of the Transportation Research Board, vol. 2003, no. 1, pp. 41-49, 2007.

[4] S. Bekhor, L. Reznikova, and T. Toledo, "A path-based algorithm for the cross-nested logit stochastic user equilibrium traffic assignment," Computer-Aided Civil and Infrastructure Engineering, vol. 24, no. 1, pp. 15-25, 2008.

[5] M. Du, H. Tan, and A. Chen, "A faster path-based algorithm with Barzilai-Borwein step size for solving stochastic traffic equilibrium models," European Journal of Operational Research, vol. 290, no. 3, pp. 982-999, 2021.

[6] W. J. Hu and X. Z. Zhou, "Multi-user and multi-mode stochastic user equilibrium model based on cross-nested logit," China Journal of Highway and Transport, vol. 25, no. 4, pp. 133-140, 2012.

[7] E. Avineri and J. N. Prashker, "Violations of expected utility theory in route-choice stated preferences," Transportation Research Record: Journal of the Transportation Research Board, vol. 1894, no. 1, pp. 222-229, 2004.

[8] C. Jie, L. A. Prashanth, M. Fu, S. Marcus, and C. Szepesvari, "Stochastic optimization in a cumulative prospect theory framework," IEEE Transactions on Automatic Control, vol. 63, no. 9, pp. 2867-2882, 2018.

[9] C. Zhang, T.-L. Liu, H.-J. Huang, and J. Chen, "A cumulative prospect theory approach to commuters' day-to-day routechoice modeling with friends' travel information," Transportation Research Part C: Emerging Technologies, vol. 86, pp. 527-548, 2018.

[10] H. K. Lo, X. W. Luo, and B. W. Y. Siu, "Degradable transport network: travel time budget of travelers with heterogeneous risk aversion," Transportation Research Part B: Methodological, vol. 40, no. 9, pp. 792-806, 2006.

[11] H. Shao, W. K. Lam, and M. L. Tam, "A reliability-based stochastic traffic assignment model for network with multiple user classes under uncertainty in demand," Networks \& Spatial Economics, vol. 6, no. 3-4, pp. 173-204, 2006.

[12] B. W. Y. Siu and H. K. Lo, "Doubly uncertain transportation network: degradable capacity and stochastic demand," $E u$ ropean Journal of Operational Research, vol. 191, no. 1, pp. 166-181, 2008.

[13] R. D. Connors and A. Sumalee, "A network equilibrium model with travellers' perception of stochastic travel times," Transportation Research Part B: Methodological, vol. 43, no. 6, pp. 614-624, 2009.

[14] H. Xu, Y. Lou, Y. Yin, and J. Zhou, "A prospect-based user equilibrium model with endogenous reference points and its application in congestion pricing," Transportation Research Part B: Methodological, vol. 45, no. 2, pp. 311-328, 2011.

[15] W. Wang and H.-j. Sun, "Cumulative prospect theory-based user equilibrium model with stochastic perception errors," Journal of Central South University, vol. 23, no. 9, pp. 24652474, 2016.
[16] J. Yang and G. Jiang, "Development of an enhanced route choice model based on cumulative prospect theory," Transportation Research Part C: Emerging Technologies, vol. 47, pp. 168-178, 2014.

[17] F. Han, X.-m. Zhao, and L. Cheng, "Traffic assignment problem under tradable credit scheme in a bi-modal stochastic transportation network: a cumulative prospect theory approach," Journal of Central South University, vol. 27, no. 1, pp. 180-197, 2020.

[18] H. X. Liu, X. He, and B. He, "Method of successive weighted averages (MSWA) and self-regulated averaging schemes for solving stochastic user equilibrium problem," Networks and Spatial Economics, vol. 9, no. 4, pp. 485-503, 2009.

[19] S. Sun and W. Y. Szeto, "Logit-based transit assignment: approach-based formulation and paradox revisit," Transportation Research Part B: Methodological, vol. 112, pp. 191215, 2018.

[20] S. Bekhor and J. N. Prashker, "Stochastic user equilibrium formulation for generalized nested logit model," Transportation Research Record: Journal of the Transportation Research Board, vol. 1752, no. 1, pp. 84-90, 2001.

[21] N. Jiang and C. Xie, "Computing and analyzing mixed equilibrium network flows with gasoline and electric vehicles," Computer-Aided Civil and Infrastructure Engineering, vol. 29, no. 8, pp. 626-641, 2014.

[22] D. Kahneman and A. Tversky, "Prospect theory: an analysis of decision under risk," Econometrica, vol. 47, no. 2, pp. 263-291, 1979.

[23] D. Prelec, "The probability weighting function," Econometrica, vol. 66, no. 3, pp. 497-528, 1998.

[24] H. A. Simon, "A behavioral model of rational choice," The Quarterly Journal of Economics, vol. 69, no. 1, pp. 99-118, 1955.

[25] B. Zhang, Z. C. Juan, and X. X. Lin, "Stochastic user equilibrium model based on cumulative prospect theory," Journal of Southwest Jiaotong University, vol. 46, no. 5, pp. 868-874, 2011. 